\title{
Low Energy Stages - 'Dogbone' Muon RLA
}

\author{
S.A. Bogacz \\ Center for Advanced Studies of Accelerators \\ Thomas Jefferson National Accelerator Facility \\ Newport News, VA 23606, USA.
}

A conceptual design of lower energy stages of muon accelerator is presented. The scheme is based on two superconducting, $200 \mathrm{MHz}$ linacs: a single pass linear pre-accelerator followed by a multi-pass 'dogbone' recirculating linac (RLA). In the presented scenario, acceleration starts after ionization cooling at $273 \mathrm{MeV} / \mathrm{c}$ and proceeds to $5 \mathrm{GeV}$, where the beam is injected into a complex of FFAG rings for further acceleration. The key conceptual issues are addressed and implemented in the overall acceleration scheme: capture, acceleration, transport and preservation of large phase space of fast decaying species. Beam transport of large-momentum-spread beams is facilitated through appropriate lattice design choices. The proposed linear optics for 'droplet' return arcs optics is further supplemented with a sextupole correction to suppress chromatic effects contributing to the horizontal emittance dilution.

\section{MUON ACCELERATION SCHEME}

A neutrino factory [1] is aimed to produce narrow neutrino beams via decay of muons in long straight sections of a storage ring. Recent 'revival' of FFAG rings for muon acceleration makes them more cost effective (compared to the RLA), but only at energies above $5 \mathrm{GeV}$. Here we propose a lower energy stage of a muon accelerator complex based on a 'dogbone' multi-pass linac. As illustrated schematically in Figure 1, a proposed muon accelerator features a $273 \mathrm{MeV} / \mathrm{c}$ to- $1.5 \mathrm{GeV}$ straight preaccelera- tor linac and a 1.5-to-5 GeV 3.5-pass recirculating linac accelerator (RLA).

The pre-accelerator captures a large muon phase space coming from the cooling channel and accelerates muons to relativistic energies of about $1.5 \mathrm{GeV}$. It makes the beam sufficiently relativistic and adiabatically decreases the phase-space volume, so that effective acceleration in recirculating linacs is possible. The 3.5-pass 'dogbone' RLA further compresses and shapes-up the longitudinal and transverse phase spaces, while increasing the energy.

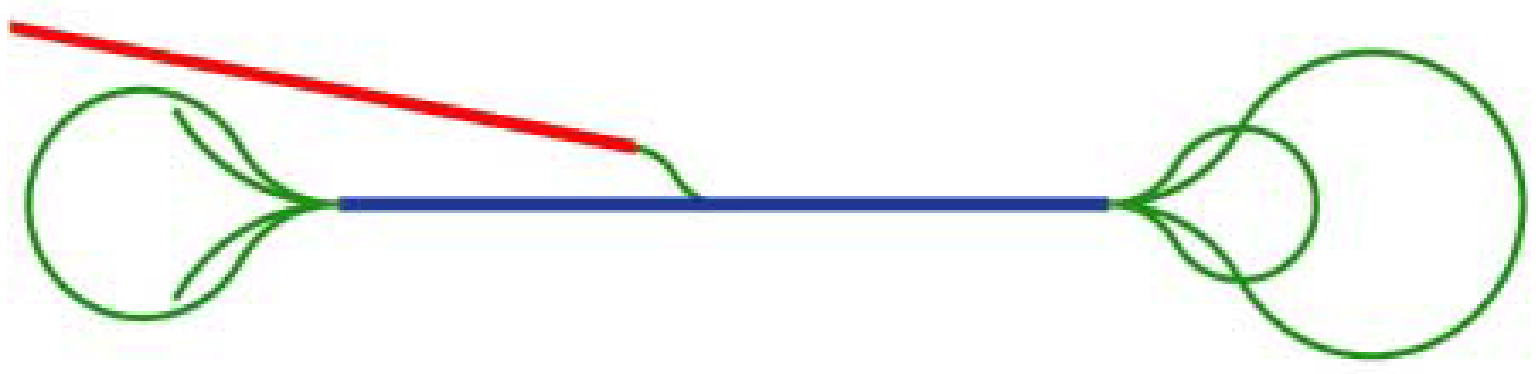

Figure 1. Low energy stages of the muon accelerator complex $-273 \mathrm{MeV} / \mathrm{c}$ to $1.5 \mathrm{GeV}$ linear pre-accelerator (red) followed by a 3-5-pass 'dogbone' RLA based on a $1 \mathrm{GeV} /$ pass main linac (blue) and 3 'droplet' return $\operatorname{arcs}($ green). 


\subsection{Accelerating Technology}

To ensure adequate survival rates of short-lived muons, acceleration must occur at high average gradient. Initial estimate [2] shows that an RF gradient of $15 \mathrm{MV} / \mathrm{m}$ will allow survival of about $85 \%$ of source muons throughout the RLA. Since muons are generated as a secondary beam they occupy large phasespace volume. The accelerator must provide high average gradient, while maintaining very large transverse and longitudinal accelerator acceptances. The above requirement drives the design to low RF frequency, e.g. $200 \mathrm{MHz}$. If normal-conducting cavities at that frequency were used, the required high gradients would demand unachievably high peak power RF sources. The RF power can then be delivered to the cavities over an extended time, and thus RF source peak power can be reduced.

\section{BEAM DYNAMICS CHOICES}

The initial longitudinal acceptance of the linear accelerator $(2.5 \sigma)$, as set by the best performance promised by the cooling channels is summarized in Table 1.

Table 1

Beam emittance after cooling at $273 \mathrm{MeV} / \mathrm{c}$ (rms)

\begin{tabular}{lc}
\hline $\begin{array}{l}\text { Transverse emittance : } \\
\varepsilon_{\mathrm{x}}, \varepsilon_{\mathrm{y}} \text { (normalized) }\end{array}$ & $4.8 \mathrm{~mm} \cdot \mathrm{rad}$ \\
\hline $\begin{array}{l}\text { longitudinal emittance: } \\
\varepsilon_{\digamma}=\sigma_{\Delta \mathrm{p}} \sigma_{\mathrm{z}} / \mathrm{m}_{\mu} \mathrm{c}\end{array}$ & $27 \mathrm{~mm}$ \\
\hline momentum spread: $\sigma_{\Delta \mathrm{p} / \mathrm{p}}$ & 0.07 \\
\hline bunch length: $\sigma_{\mathrm{z}}$ & $176 \mathrm{~mm}$ \\
\hline
\end{tabular}

\subsection{Transverse Dynamics}

In a recirculating linac one needs to separate different energy beams coming out of a linac and to direct them into appropriate arcs for recirculation. For multiple practical reasons horizontal rather than vertical beam separation was chosen [2]. Rather than suppressing horizontal dispersion created by the spreaders and recombiners it is smoothly matched to the horizontal dispersion of the return arcs. Finally, to assure compact arc architecture very short matching sections in spreaders and recombiners are desired. in spreaders and recombiners are desired. One also needs to maintain manageable beam sizes in the arcs [3]. As illustrated in Figure 4, this calls for short cells and for putting stringent limits on dispersion and beta functions (beam envelope). Since spreaders and recombiners were chosen in the horizontal plane, the uniform focusing and lattice regularity was broken in that plane and the horizontal beam envelope requires special attention. On the other hand, the vertical beam size remains small due to maintaining uniform focusing and small beta functions in that plane.

\subsection{Longitudinal Dynamics}

The initial longitudinal acceptance of the linear accelerator is chosen to be $2.5 \sigma$, i.e. $\Delta p / p= \pm 17 \%$ and $\mathrm{RF}$ pulse length $\Delta \phi= \pm 62$ deg. To perform adiabatic bunching, the RF phase of the cavities is shifted by 72 deg at the beginning of the pre-accelerator and gradually changed to zero by the linac end. In the first half of the linac, when the beam is still not sufficiently relativistic, the offset causes synchrotron motion, allowing bunch compression in both length and momentum spread to $\Delta p / p= \pm 7 \%$ and $\Delta \phi= \pm 29 \mathrm{deg}$. The synchrotron motion also suppresses the sag in acceleration for the bunch head and tail. Figure 3 shows how the initially elliptical boundary of the bunch longitudinal phase space will be transformed by the end of the linac.

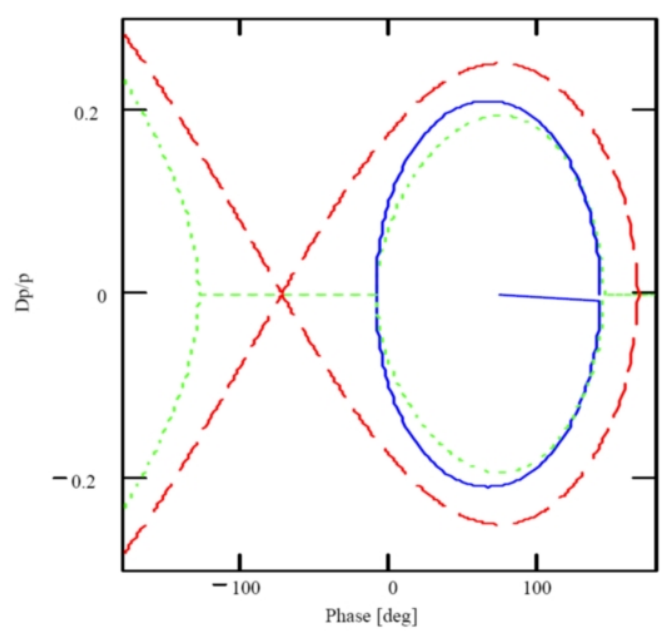

Figure 2. Longitudinal beam boundary (solid line) inside a separatrix (dashed line) shown at the beginning of the linac $(\Delta \mathrm{p} / \mathrm{p}= \pm 0.17$ or $\Delta \phi= \pm 62 \mathrm{deg}$. $)$ 
Fri Dec 03 09:03:52 2004 OptiM - MAIN: - D:IStudy 2AIPreLinaclLinac_sol.op
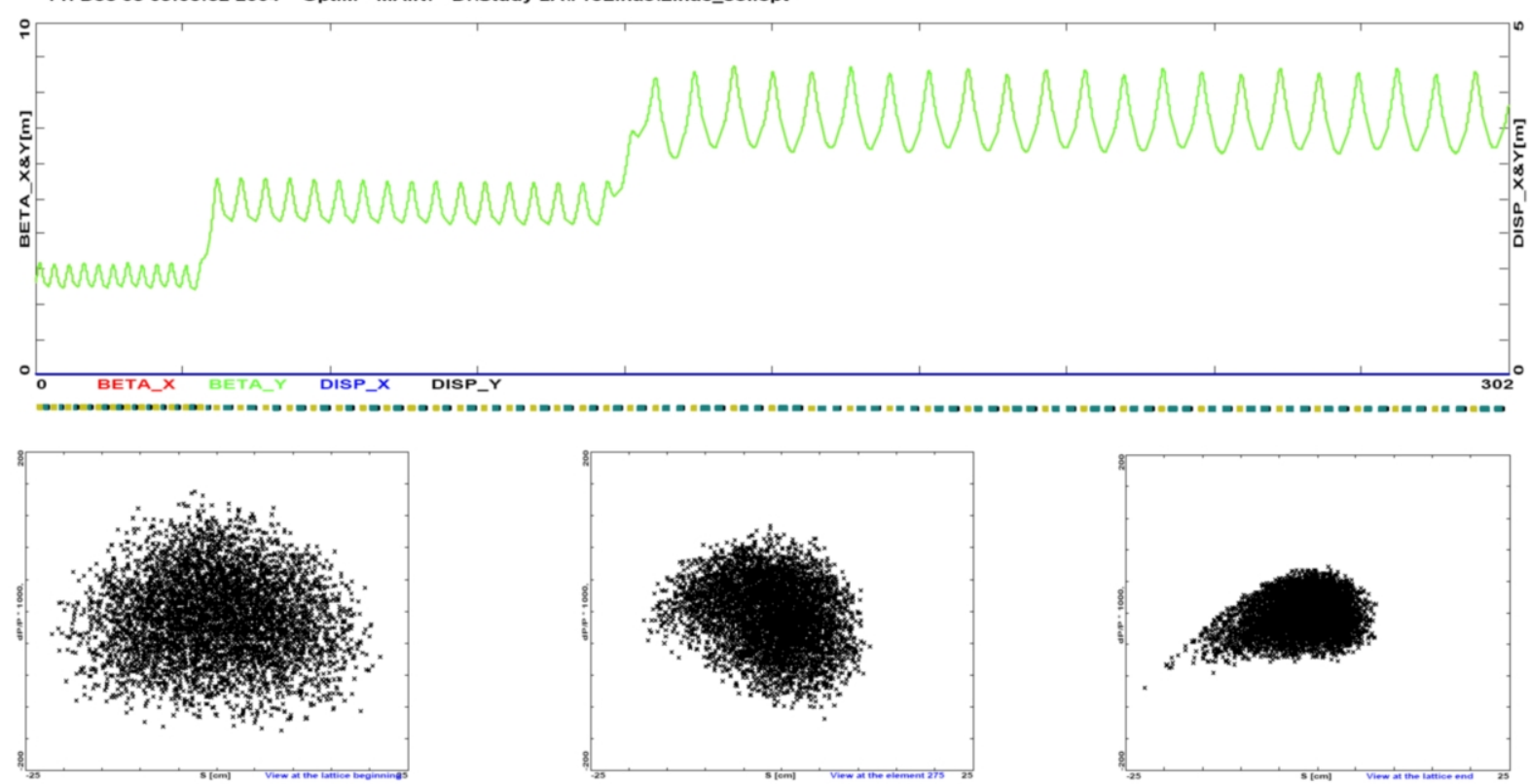

Figure 3. Top, transverse optics of the pre-accelerator linac - uniform periodic focusing with three styles of cryomodules. Below, longitudinal phase-space: before, half-way through and at the end of acceleration, as illustrated by particle tracking.

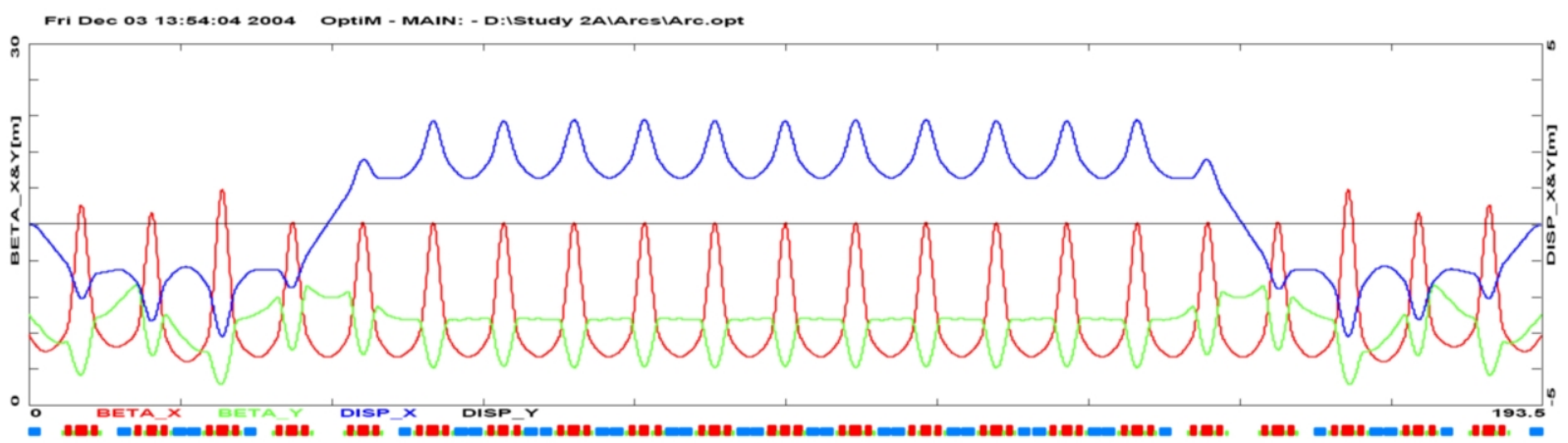

Figure 4. 'Droplet' return arc - transverse optics featuring periodic beta-functions and the horizontal dispersion matched to two consecutive passes through the main linac

\subsection{Multi-pass Linac Optics}

To maintain uniform focusing periodicity between linacs and arcs one needs to minimize Twiss functions for multi-pass optics of both linacs. This can be achieved through designing a semi-periodic linac optics (introducing additional large scale lattice period). As illustrated in Figures 5, resulting Twiss functions (at linac ends) are significantly lowered, which facilitates uniform matching between different types of optics to alleviate emittance dilution due to chromatic aberrations. Finally, there is a need for suppression of chromatic effects via sextupole corrections in spreaders and recombiners. This was implemented via three families of sextupoles [4] to control the horizontal emittance blow-up. 

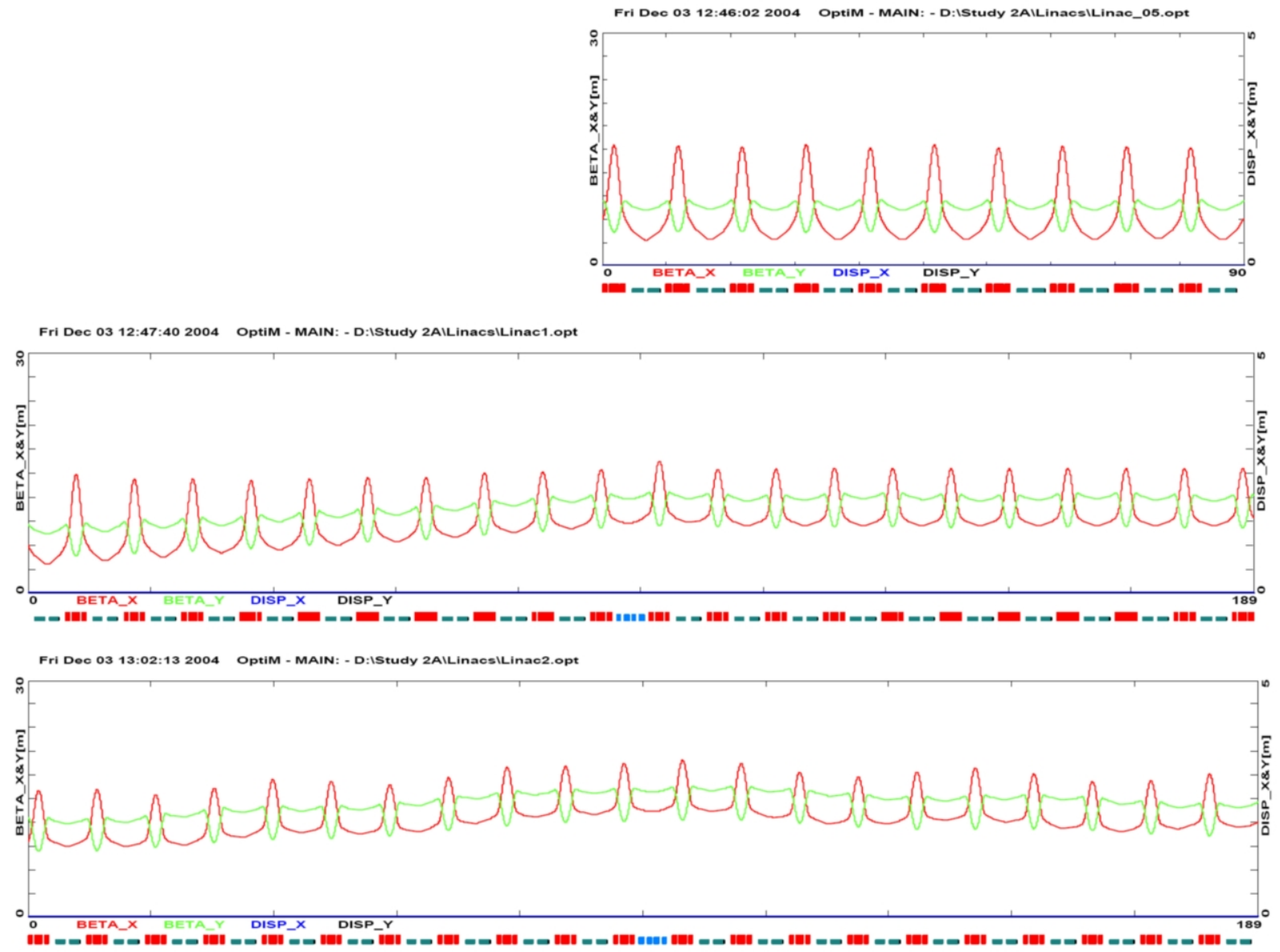

Figure 5. Optimized multi pass linac optics - uniform periodic focusing lattice for: $1.5-2 \mathrm{GeV}, 2-3 \mathrm{GeV}$ and 3-

$4 \mathrm{GeV}$ passes.

\section{CONCLUSIONS}

Results of this study suggest that there are no obvious physical or technical limitations precluding construction of a 'dogbone' RLA for acceleration of muons to $5 \mathrm{GeV}$. The proposed acceleration and beam transport scheme is well suited for handling large phase space beams. Proposed in the Study II RLA chromatic corrections via two families of sextupoles [4] should to be very effective in emittance dilution control for this scheme as well.

\section{REFERENCES}

1. M.M. Alsharo's et al., Physical Review Special Topics - Accelerators and Beams, Vol 6, 081001 (2003)

2.D. Douglas and V.A. Lebedev, Nuclear Instruments and Methods in Physics Research A, Vol 472, 499, (2001)
3. S.A. Bogacz and V.A. Lebedev, Nuclear Instruments and Methods in Physics Research A, Vol 503, 306, (2003)

4. S.A. Bogacz and V.A. Lebedev, Nuclear Instruments and Methods in Physics Research A, Vol 472, 528, (2001) 